\title{
OSSERVAZIONI SULLE SCALE SEMANTICHE IN ITALIANO A COFRONTO DELL'INGLESE E DEL CECO ${ }^{1}$
}

\section{Introduzione}

Nell'ambito della teoria della prospettiva funzionale dell'enunciato (PFE) quest'ultimo è concepito come il campo comunicativo in cui gli elementi rispettivi di esso rappresentano diverse unità comunicative. ${ }^{2}$ Queste unità comunicative si differenziano a seconda del grado di dinamismo comunicativo (DC). ${ }^{3}$ Il grado di DC è il risultato dell'interazione dei quattro fattori della PFE: ordinamento lineare, ovvero l'ordine dei costituenti, contesto, struttura semantica e, per la lingua parlata intonazione. ${ }^{4}$ In dipendenza del grado di DC, un elemento ha la funzione di una delle unità comunicative dell'enunciato, cioè quella di tema, di transizione o di rema. $^{5}$

Nelle lingue naturali, i suddetti fattori si presentano come indicatori della prospettiva comunicativa dell'enunciato in maniera diversa, eppure sempre in interazione fra di loro. Secondo Firbas è il contesto ad essere preso in considerazione prima di tutto. ${ }^{6}$ Ad illustrare ciò servirà l'esempio che segue: ${ }^{7}$

\footnotetext{
${ }^{1}$ Nelle nostre osservazioni ci avvaliamo delle argomentazioni di due articoli pubblicati a proposito delle scale semantiche: quello di Jana CHAMONIKOLASOvá Dynamic semantic scales in the theory of functional sentence perspective, e quello di Aleš Svoboda Firbasian Semantic Scales and Comparative Studies. Infatti, questi due articoli sono considerabili come una sintesi della teoria delle funzioni semantiche dei costituenti dell'enunciato sulle scale semantiche di Firbas e creano, a questo proposito, un'affidabile base. Per il concetto delle scale semantiche e la loro struttura cfr. parte 2 . di questo articolo.

${ }^{2}$ La teoria della PFE è stata riassunta da Jan FIRBAS nel suo libro intitolato Functional sentence perspective in written and spoken communication, Cambridge 1992.

${ }^{3}$ «By the degree of CD carried by a sentence element we understand the extent to which the sentence element contributes to the development of the communication, to which, as it were, it 'pushes' the communication forward. The elements carrying the lowest degree of CD constitute the theme, those carrying the highest degree, the rheme». FIRBAS $(1966: 240)$.

${ }^{4}$ «In assessing degrees of $\mathrm{CD}$, the analyses of the written texts have taken into consideration (i) linear modification, (ii) the character of the semantic content of the linguistic element as well as the character of the semantic relations involved, and (iii) the retrievability of the information from the immediately relevant preceding context. An interplay of these three factors determines the distribution of degrees of $\mathrm{CD}$ over the written sentence. ... It determines its functional sentence perspective». FIRBAS (1992: 10).

${ }_{6}^{5}$ Per la lista completa delle unità comunicative cfr. SvOBODA (1989: 27 e 31).

${ }^{6}$ «Within the interplay of means, the leading role is played by context. It is context, strictly speaking the ad-hoc situational and the preceding verbal context (...), that determines the extent to, and the manner in, which semantic structure and linearity are allowed to come into play. This is in agreement with the observation that a semantic and grammatical sentence structure becomes a genuine sentence only when serving a definite communicative purpose, i.e. within a definite context» FIRBAS (1979 : 45).

${ }^{7}$ La lista delle fonti degli esempi viene data dopo la bibliografia. Nelle versioni rispettive, l'elemento rematico è in corsivo.
} 
(1) Entrò Carla.

Carla came into the room.

Vstoupila Karla.
[IND p. 1]

[TIM p. 5]

Con questa frase, la prima del romanzo Gli indifferenti di A. Moravia, viene introdotto «sulla scena » uno dei protagonisti della storia, cioè Carla ${ }^{8}$ Essendo la prima frase del romanzo, non possiamo appoggiarci a nessun contesto linguistico. Nella versione italiana e in quella ceca, il soggetto rematico Carla/Karla occupa la posizione finale nell'enunciato, mentre nella versione inglese tale elemento si osserva nella posizione iniziale. Vale a dire che nella versione italiana e in quella ceca sono il contesto e l'ordinamento lineare a segnalare il grado di DC degli elementi dell'enunciato, mentre nella versione inglese è solo il contesto a indicarlo. La differenza fra l'ordinamento lineare italiano e ceco da una parte e quello inglese dall'altra parte è dovuta all'impatto delle regole grammaticali vigenti nelle rispettive lingue.

Per quanto riguarda l'ordine dei costituenti, il ceco e l'inglese sono considerabili poli opposti. In ceco, grazie alla flessione nominale e quella verbale, si osserva l'ordine dei costituenti quasi libero, con una tendenza a mettere verso la fine della frase quell'elemento che funge da rema. In inglese, invece, grazie all'assenza della flessione nominale, si osserva l'ordine grammaticalizzato, cioè fisso $S-V-O$. La sua funzione è quella di indicare le relazioni sintattiche dei rispettivi elementi della frase e non quella di segnalare le loro funzioni comunicative.

L'ordine dei costituenti nella frase italiana è a metà strada tra il modello ceco e quello inglese. Da una parte, grazie all'assenza della flessione nominale, l'ordine $S-V-O$ è considerato come sintatticamente non marcato, dall'altra parte, però, grazie alla flessione verbale e quella pronominale anche in italiano esiste la possibilità di modificarlo. ${ }^{9}$

Così per il ceco si può constatare una forte e per l'italiano abbastanza forte tendenza alla cosiddetta distribuzione fondamentale di DC per cui la regola di base è: più vicino alla fine dell'enunciato, più alto il grado di DC del costituente.

Finora sono stati presi in considerazione due fattori della PFE, cioè l'ordinamento lineare e il contesto. Nella versione italiana e in quella ceca dell'esempio (1) questi due fattori sono sufficienti per segnalare la prospettiva comunicativa dell'enunciato. Il soggetto è identificato come rematico non solo per la sua posizione ma anche per essere non ricavabile dal contesto. Nella versione inglese, invece, né l'ordinamento lineare né il contesto segnalano in modo univoco la prospettiva comunicativa dell'enunciato. Infatti, la frase «Carla entered into the room », con l'ordine $S$ - $V$, può essere soggetta a più interpretazioni. Fuori contesto tutti i suoi elementi possono avere la funzione rematica. Questa frase può funzionare da risposta a diverse domande: alla domanda «Who came? », alla domanda «What did Carla do » ma anche come risposta alla domanda «Where did Carla come? ». Quindi possiamo concludere che la frase inglese è inseribile in

\footnotetext{
${ }^{8}$ Per la struttura, l'esempio corrisponde alla frase di Firbas, oggi considerabile classica: «A boy came into the room». FIRBAS $(1992: 59)$.

${ }^{9}$ Cfr. per esempio KLÍMOvÁ (2002).
} 
tre contesti diversi, naturalmente con diversi schemi di intonazione. ${ }^{10}$ In questo momento è il terzo fattore della PFE ad assumere rilevanza, cioè la struttura semantica. Il soggetto non ricavabile dal contesto nella posizione iniziale viene introdotto come «fenomeno presentato sulla scena». Vale a dire, il soggetto Carla è considerabile come «nuovo » grazie alla sua funzione semantica. Si può quindi constatare che, nella versione inglese, sono il contesto e la struttura semantica a cooperare nell'indicazione del soggetto come rema, mentre nella versione italiana e in quella ceca, svolge tale funzione il contesto e l'ordinamento lineare.

Successivamente verrà preso in considerazione l'ordinamento degli elementi dell'enunciato in italiano, in inglese e in ceco sulle scale semantiche di Firbas, cioè sulla scala presentativa e sulla scala di qualità, stabilite in dipendenza delle funzioni semantiche che essi svolgono. ${ }^{11}$ Dal confronto tra l'italiano, l'inglese e il ceco può emergere come le caratteristiche morfosintattiche di lingue tipologicamente diverse influenzino il modo d'indicazione delle funzioni semantiche degli elementi della frase, ma anche come tutti i fattori della PFE si presentino nella prospettiva comunicativa dell'enunciato.

\section{Scale semantiche e funzioni semantiche}

La struttura semantica è il fattore della PFE che si rivela decisivo soprattutto nei casi in cui il contesto e l'ordinamento lineare non sono sufficienti all'individuazione della funzione comunicativa degli elementi dell'enunciato (cfr. la versione inglese dell'esempio (1)). Sulla scala presentativa la comunicazione è orientata verso il soggetto grammaticale, mentre sulla scala di qualità la comunicazione è orientata verso un elemento diverso da esso. ${ }^{12}$ Vale a dire che su queste due scale semantiche il soggetto, come tutti gli altri componenti dell'enunciato, svolge funzioni semantiche diverse. Ad evidenziarlo sono lo schema della scala presentativa e quello della scala di qualità con le funzioni semantiche e con le unità comunicative rispettive:

\section{Scala presentativa}

\begin{tabular}{|c|c|c|c|c|}
\hline $\begin{array}{c}\text { Scena (Sc) } \\
\qquad \\
\downarrow \\
\text { Tema (T) }\end{array}$ & \multicolumn{2}{|c|}{$\begin{array}{c}\text { Presentazione } \\
\text { sulla scena (Pr) } \\
\downarrow \\
\text { Transizione (Tr) } \\
\end{array}$} & \multicolumn{2}{|c|}{$\begin{array}{c}\text { Fenomeno } \\
\text { presentato sulla scena (F) } \\
\downarrow \\
\qquad \\
\text { Rema }(\boldsymbol{R})\end{array}$} \\
\hline $\begin{array}{l}\text { (2) } \mathrm{C}^{\prime} \\
\text { There } \\
(\mathrm{T})\end{array}$ & $\begin{array}{l}\text { era } \\
\text { was } \\
\mathrm{Tr}\end{array}$ & $\begin{array}{l}\text { un odore pesante } \\
\text { a heavy odour } \\
\qquad R\end{array}$ & $\begin{array}{l}\text { nella stanza } \\
\text { in the room } \\
\text { T }\end{array}$ & $\begin{array}{l}{[\mathrm{GEN} \text { p. 31] }} \\
\text { [DUB p. 6] }\end{array}$ \\
\hline $\begin{array}{l}\text { V místnosti } \\
\mathrm{T}\end{array}$ & $\begin{array}{l}\text { byla } \\
\text { Tr }\end{array}$ & $\begin{array}{c}\text { těžká vůně } \\
R\end{array}$ & & \\
\hline
\end{tabular}

${ }^{10}$ Cfr. KLímová (2002 : 44).

${ }^{11}$ Cfr. Firbas (1992 : 66-67).

${ }^{12}$ Cfr. Chamonikolasová (2005: 61). 
Nella versione italiana e in quella inglese dell'esempio (2), il soggetto rematico un odore pesantela heavy odour nella posizione postverbale è il «Fenomeno presentato sulla scena». Il verbo era/was è la transizione esprimendo «Presentazione sulla scena ». La struttura avverbiale nella stanza/in the room che, nonostante la posizione finale, è un elemento tematico, con la funzione di «Scena». È anticipata dalla particella avverbiale ci/there che permette l'ordinamento $V$-S anche in inglese. Nella versione ceca, la « Scena »v místnosti si osserva nella posizione non marcata all'inizio della frase. Bisogna aggiungere che, in inglese, l'ordine $V-S$ viene considerato come normale solo nella struttura presentativa there is/there are, mentre in italiano è normale nelle frasi con un soggetto rematico (cfr. l'esempio (1) e anche gli esempi (4) e (5) più avanti).

\section{Scala di qualità}

\begin{tabular}{|cccc|}
\hline $\begin{array}{c}\text { (Scena) } \\
\downarrow\end{array}$ & \multicolumn{1}{c}{ Portatore di qualità $(\mathbf{P})$} & Qualità $(\mathbf{Q})$ & Specificazione/-i $(\mathrm{Sp})$ \\
& $\downarrow$ & $\downarrow$ & $\downarrow$ \\
Tema $(\mathrm{T})$ & Transizione $(\mathrm{Tr})$ & $\operatorname{Rema}(\boldsymbol{R})$ \\
\hline
\end{tabular}

(3) Nel corridoio In the corridor she $\mathrm{V}$ předsíni $\mathrm{T}$

incontrò
met
potkala

$\operatorname{Tr}$

$\begin{array}{ll}\text { Michele } & \text { [IND p. 12] } \\ \text { Michele } & \text { [TIM p. 12] } \\ \text { Michela } & \\ R & \end{array}$

Nella frase (3), che rappresenta la realizzazione della scala di qualità, la comunicazione è orientata verso la specificazione. In altre parole, il complemento oggetto Michele nella posizione finale svolge la funzione semantica di specificazione e quindi è il rema dell'enunciato. Il costituente nel corridoio/in the corridor/v predsíni, in tutte e tre le versioni nella posizione iniziale, fa parte del contesto situazionale e, fungendo da «Scena», fa parte del tema. Fa parte del tema anche il soggetto grammaticale indicato, in italiano e in ceco, dalla desinenza verbale $-\grave{o} /-a$, in inglese dal pronome she. La sua funzione semantica è quella di «Portatore di qualità ». Il verbo incontrò/met/potkala è la transizione e funge la «Qualità ».

L'ordinamento degli elementi della frase sulle scale semantiche riflette un costante aumento del grado di DC, ovvero riflette l'andamento dall'elemento che ne porta il grado minimo all'elemento che ne porta il grado massimo, corrispondendo così alla distribuzione fondamentale di DC.

\subsection{Scala presentativa}

L'esempio (1) è stato usato come punto di partenza non solo per presentare i fattori della PFE. È stato usato anche con lo scopo di evidenziare che in diverse lingue gli elementi possono essere organizzati sulla scala presentativa in modo diverso, e che l'ordine delle parole non deve necessariamente seguire quell'andamento dall'elemento che porta il grado minimo di DC all'elemento che ne porta il grado massimo. Nell'esempio successivo si osserva lo stesso ordinamento lineare e lo stesso verbo come nell'esempio (1). In questo caso, però, la frase è inserita in un contesto: 
(4) Entrarono due servitori, ...

[GATT p. 71]

Two lackeys entered, ...

[LEOP p. 46]

Vstoupili dva sloužící, ...

Nella versione italiana e in quella ceca l'ordine dei costituenti è $V$-S, a differenza della versione inglese con l'ordine $S$ - $V$. Il soggetto due servitori/two lackeys/dva sloužící non è ricavabile dal contesto, perciò funge da «Fenomeno presentato sulla scena ». Quindi si può concludere che in italiano e in ceco, come nell'esempio (1), sono il contesto e l'ordinamento lineare a cooperare nell'atto di indicare il soggetto come rema, mentre in inglese sono il contesto e la struttura semantica a confermarlo come tale. (Quest'esempio è stato usato anche con lo scopo di anticipare alcuni esempi della sezione di questo articolo, in cui vengono osservate le funzioni semantiche degli elementi della frase sulla scala di qualità.)

Nell'esempio successivo, una differenza si registra non solo tra l'italiano e l'inglese, ma anche tra l'italiano e il ceco:

(5) E nei giorni normali nella vetrina era appeso un avviso ...

[GEN p. 29]

And on ordinary days $\boldsymbol{a}$ notice used to hang in the window ... [DUB p. 3]

Ve všední dny ve vitríně visela cedulka ...

Nella versione italiana e in quella ceca, l'ordine $\mathrm{C}_{t^{-}} C_{l^{-}}-\mathrm{V}-\mathrm{S}$ corrisponde alla distribuzione fondamentale di DC: ${ }^{13}$ il soggetto rematico un avviso/cedulka occupa la posizione finale e funge da «Fenomeno presentato sulla scena ». In italiano, è l'articolo indeterminativo, oltre alla posizione, a confermarlo in questa funzione. Nella versione inglese l'ordine è $\mathrm{C}_{t}-V-S-C_{l}$ : il soggetto è indicato in funzione di «Fenomeno » solo dall'articolo indeterminativo. Così si può concludere che, ad indicare il soggetto come rema, in ceco è l'ordinamento lineare, in inglese è uno strumento grammaticale, mentre in italiano entrambi possono svolgere questa funzione.

Nell'esempio (4) e nell'esempio (5), nelle versioni italiane e in quelle ceche, si osservano tre fattori della PFE in interazione: il contesto, l'ordinamento lineare e la struttura semantica, mentre nella versione inglese ne sono presenti solo due: il contesto e la struttura semantica. L'esempio che segue ha lo scopo di evidenziare che, anche in italiano, la realizzazione della scala presentativa può risultare dall'interazione di questi due fattori. Vale a dire che anche in italiano esistono delle frasi in cui l'ordinamento lineare non corrisponde alla distribuzione fondamentale di DC, cioè frasi in cui la funzione semantica del soggetto è indicata solo dall'articolo, esattamente come in inglese:

(6) Un mazzo di crespo era legato al battente della porta con un nastro.

A crape bouquet was tied to the door-knocker with ribbon.

[GEN p. 29]

[DUB p. 3]

Ke klepátku na dveřích byla stužkou přivázána kytka z krepového papíru.

Con l'ordinamento $S$ - $V$ sia nella versione italiana che nella versione inglese, la funzione semantica del soggetto un mazzo di crespo/a crape bouquet è segnalata

${ }^{13}$ Per $\mathrm{C}_{\mathrm{l}}$ si intende complemento di luogo, per $\mathrm{C}_{\mathrm{t}}$ s'intende complemento di tempo. 
dall'articolo indeterminativo, mentre nella versione ceca è segnalata dall'ordinamento $V$-S. L'azione dell'articolo diventa evidente se prendiamo in considerazione una variante di questa frase in cui invece dell'articolo indeterminativo appare quello determinativo:

(7) Il mazzo di crespo era legato al battente della porta con un nastro.

The crape bouquet was tied to the door-knocker with ribbon.

Kytka z krepového papíru byla ke klepátku na dveřích přivázána stužkou.

Nella versione italiana e in quella inglese, l'articolo determinativo indica il soggetto come ricavabile dal contesto. Di conseguenza, rispetto all'esempio (6), cambia la funzione semantica del soggetto e anche la prospettiva comunicativa dell'enunciato: invece del soggetto rematico in funzione di «Fenomeno » sulla scala presentativa il soggetto diventa tema e funge da «Portatore di qualità » sulla scala di qualità.

\subsection{Scala di qualità}

Per l'analisi degli enunciati rappresentanti la realizzazione della scala di qualità, viene presa, come punto di partenza, l'affermazione che su questa scala la comunicazione è orientata verso un elemento diverso dal soggetto grammaticale. (Per le funzioni semantiche di tutti gli altri elementi della frase vedi sopra lo schema della scala di qualità.)

I primi due esempi di questa sezione sono anticipati dall'esempio (4) usato con lo scopo di evidenziare che lo stesso verbo può, in dipendenza della struttura semantica dell' enunciato, esercitare funzioni diverse:

Nella frase

(8) (Don Fabrizio ... si affrettò a uscire dal bagno ...) prima che padre Pirrone entrasse.

[GATT p. 72]

(Don Fabrizio ... hurried to leave the bath ...) before the Jesuit entered.

[LEOP p. 46]

(Don Fabrizio ... spěchal, aby byl venku z vany,) dřív než otec Pirrone vstoupí.

la realizzazione dello schema della scala di qualità viene ridotta al minimo di due unità: «Portatore di qualità » e «Qualità ». Dal confronto emerge che tra la versione italiana, inglese e quella ceca non è registrabile nessuna differenza: il soggetto tematico padre Pirrone/the Jesuit/otec Pirrone con la funzione di «Portatore di qualità » occupa la posizione davanti al verbo rematico entrasse/entered/vstoupí che funge da «Qualità ». Lo stesso verbo, con la stessa funzione è osservabile nella frase

(9) (... annunziavano ... che) si entrava nelle terre di casa Salina.

[GATT p. 60]

(... a message that) they were entering the family estates. [LEOP p. 35]

(... oznamovaly ..., že) vstupují na pozemky Salinů.

In questo caso, però, un altro componente della scala di qualità è presente, cioè la « Specificazione » e funge quindi da rema dell'enunciato. Per quanto riguarda la realizzazione della scala semantica, tra le tre versioni non c'è nessuna differenza. 
Tuttavia c'è una differenza da menzionare riguardo alla struttura sintattica della frase, fra la versione originale e quella ceca da una parte, e la frase inglese dall'altra. Mentre nella versione italiana e in quella ceca la specificazione nelle terre di casa Salina/na pozemky Salinu svolge la funzione sintattica di complemento di luogo, nella versione inglese la specificazione the family estates è il complemento oggetto. Questa differenza, però, non si riflette nella prospettiva comunicativa dell'enunciato.

Le differenze che si registrano, tra la versione italiana e quella inglese sono notevoli nell'esempio successivo:

... e magari qualche compenso lo avranno.

[GATT p. 46]

...; they may even get some sort of compensation.

... a nějakou kompenzaci snad dostanou. ${ }^{14}$

[LEOP p. 26]

Nella versione italiana, l'ordinamento $O_{d^{-}}$(pronom)- $V_{(S)}$ rappresenta la cosiddetta dislocazione a sinistra: il complemento oggetto compenso è dislocato nella posizione iniziale, mentre la sua funzione sintattica è indicata dalla forma di accusativo del pronome lo. Così il complemento oggetto funge da «Scena » e il soggetto grammaticale, indicato dalla desinenza verbale -anno, svolge la funzione di « Portatore di qualità ». È il verbo avranno, in funzione di « Qualità », a fungere da rema. Nella versione ceca, l'ordinamento $O_{d^{-}} V_{(S)}$ corrisponde a quello della versione italiana, con il verbo come rema. Nella versione inglese l'ordine è $S-V-O_{d}$, le funzioni semantiche sono però uguali. In un caso del genere, nella lingua parlata, il verbo sarebbe rematizzato tramite l'intonazione, cioè diventando il centro d'intonazione. In altre parole, sarebbe l'intonazione, come fattore della PFE, a indicare la funzione rematica del verbo. Dunque si può concludere che la differenza tra l'ordinamento lineare della frase italiana e ceca da una parte e quello della frase inglese dall'altra parte non esercita nessuna influenza sull'attribuzione delle funzioni semantiche e perciò neanche sulla distribuzione di DC.

\section{Conclusione}

Le differenze tra l'italiano, l'inglese e il ceco osservabili nelle realizzazioni delle scale semantiche e nell'atto di indicare le rispettive funzioni semantiche, sono dovute all'impatto delle regole grammaticali vigenti in queste tre lingue.

In italiano, in riferimento alla scala presentativa, si attestano due realizzazioni diverse: a) l'ordinamento $S$ - $V$, in cui il soggetto grammaticale viene indicato come rematico dall'articolo indeterminativo, b) l'ordinamento $V-S$, con o senza la struttura presentativa c'è/ci sono (cfr. l'esempio (1) e (4)). Anche nella realizzazione della scala di qualità, grazie alla flessione pronominale e a quella verbale, è registrabile una tendenza a mettere verso la fine della frase l'elemento rematico. In questo senso, nelle realizzazioni delle scale semantiche non si osservano notevoli differenze tra l'italiano e il ceco, puramente flessionale. Così si può concludere che nelle realizzazioni delle scale semantiche si ottiene, in entrambe le lingue, la distribuzione fondamentale di DC, come risultato dell'interazione tra il contesto e l'ordinamento lineare.

\footnotetext{
${ }^{14}$ Cfr. la traduzione ceca con l'ordine $S$ - $V$ - $O$ «A dokonce dostanou nějaké odškodnění » in LampedusA, di, G. T. (2004 $)$, Gepard, (traduzione di Jaroslav Pokorný). Praha, Academia, p. 25. 
In inglese la realizzazione di tutte e due le scale semantiche è fortemente influenzata dall'ordine delle parole grammaticalizzato. Tranne che nella struttura presentativa there is/are, il soggetto occupa la posizione preverbale essendo indicato come rematico dall' articolo indeterminativo e come tematico dall' articolo determinativo. Anche se nella frase inglese non si ottiene la distribuzione fondamentale di DC, il contesto e la struttura semantica sono sufficienti per indicare le funzioni comunicative attribuite agli elementi della frase e quindi la prospettiva dell'enunciato.

\section{BIBLIOGRAFIA}

AnTINUCCI, Francesco, CinQue, Guglielmo (1977), Sull'ordine delle parole in italiano: l'emarginazione, SGI 6, Firenze, Accademia della Crusca, p. 121-147.

BECCARIA, Gian Luigi (a cura di) (1996), Dizionario di linguistica, Torino, Einaudi.

BenincÀ, Paola, SAlvi, Giampaolo, Frison, Lorenza (1991), L'ordine degli elementi della frase e le costruzioni marcate, in RENZI (1991 : 115-225).

Chamonikolasová, Jana (2005), Dynamic semantic scales in the theory of functional sentence perspective, in Aleg(r)ace pro Evu. Papers in Honour of Eva Hajičová, p. 61-67.

CRESTI, Emanuela (1977), Frase e intonazione. SGI 6, Firenze, Accademia della Crusca, p. 45-67.

DANEŠ, František (1964), A Three-Level Approach to Syntax, TLP, 1, Prague, p. 225-240.

FIRBAS, Jan (1966), Non-thematic subjects in contemporary English, TLP, 2, Prague, p. 239-256.

FIRBAS, Jan (1979), A functional view of 'ordo naturalis', Brno Studies in English, 13, p. 29-59.

FIRBAS, Jan (1991), Il funzionamento del dinamismo comunicativo nella prospettiva funzionale della frase, in SORNICOLA, SvOBODA (1991 : 194-209).

FIRBAS, Jan (1992), Functional sentence perspective in written and spoken communication, Cambridge, Cambridge University Press.

KLÍMOVÁ, Eva (2002), Dislocazione a sinistra: descrizione formale e funzionale, Sborník prací Filozofické fakulty brněnské univerzity, L 23, p. 71-79.

KLÍMOVÁ, Eva (2002), Ordinamento lineare dell'enunciato in italiano, Linguistica Pragensia XII/1, p. 40-49.

KLÍMOVÁ, Eva (2003), Italské určité sloveso z pohledu funkční větné perspektivy ve srovnání s určitým slovesem anglickým, Opava, FPF SU.

KLíMOVÁ, Eva (2004), Il verbo italiano e il verbo inglese sulle scale semantiche, Linguistica Pragensia, XIV/1, p. 32-41.

LONZI, Lidia (1974), L'articolazione presupposizione-asserzione e l'ordine V-S in italiano, in Fenomeni morfologici e sintattici nell'italiano contemporaneo, Roma, 1974, p. 197-215.

LONZI, Lidia (1985), Pertinenza della struttura Tema-Rema per l'analisi sintattica, in STAMMERJOHANN (1986: 99-120).

MATHESIUS, Vilém (1939), O tak zvaném aktuálním členění větném, Slovo a slovesnost, 5, p. 171-174. 
RENZI, Lorenzo (a cura di) (1991), Grande grammatica italiana di consultazione, vol. I, Bologna, Mulino.

SORNICOLA, Rosanna (1985), Costituenza, dipendenza e la struttura tema-rema in italiano, in STAMMERJOHANN (1986 : 121-139).

Sornicola, Rosanna, SvobodA Aleš (a cura di) (1991), Il campo di tensione, Napoli, Liguori.

STAMMERJOHANN, Hans (1986), Tema-Rema in Italiano, Tübingen.

SVOBODA, Aleš (1989), Kapitoly z funkční syntaxe, Praha, SPN.

SvobodA, Aleš (1991), Le posizioni nell'ordine delle parole ceco dal punto di vista dell' articolazione attuale, in SORNICOLA, SVOBODA (1991 : 423-452).

SvobodA, Aleš (2005), Firbasian Semantic Scales and Comparative Studies, in Čermák, J. et al. (eds.) (2005), Patterns (A Festschrift for Libuše Dušková), Praha, Filozofická fakulta Univerzity Karlovy, p. 217-229.

\title{
Fonti degli esempi
}

JOYCE James (1992), Dubliners, London, Penguin Books. [Abbr. DUB]

JoYCE James (1995), Gente di Dublino (traduzione di Marina Emo Capodilista), Luigi Reverdito Editore. [Abbr. GEN]

MorAVIA Alberto (1992), Gli indifferenti, Milano, Tascabili Bompiani. [Abbr. IND]

MORAVIA Alberto (1975), The Time of Indifference (traduzione di Angus Davidson), Frogmore, Panther. [Abbr. TIM]

TOMASI DI LAMPEDUSA Giuseppe $\left(1993^{7}\right)$, Il Gattopardo, Milano, Feltrinelli. [Abbr. GATT]

TOMASI DI LAMPEDUSA Giuseppe (1996), The Leopard (traduzione di Archibald Colquhoun), London, The Harvill Press. [Abbr. LEOP]

\begin{abstract}
The contribution deals with semantics as one of the factors of functional sentence perspective (FSP) in Italian, in comparison with English and Czech. Firstly, all the factors of FSP are introduced, i.e. linearity, context, semantics, and, in the spoken language, intonation, with the aim to show that the sentence perspective is the result of their interplay. The main attention is paid to semantics: sentence elements are observed in their dynamic semantic functions on the Firbasian presentation scale and quality scale with regard to the impact that the grammatical, i.e. morpho-syntactical rules of typologically different languages play not only in the process of the indication of dynamic semantic functions of a sentence item but also in the way in which all FSP factors may manifest themselves.
\end{abstract}

